Comment. Math. Helv. 73 (1998) 122-136

(C) 1998 Birkhäuser Verlag, Basel

$0010-2571 / 98 / 010122-15 \$ 1.50+0.20 / 0$

Commentarii Mathematici Helvetici

\title{
Groups acting on finite dimensional spaces with finite stabilizers
}

Peter H. Kropholler and Guido Mislin

\begin{abstract}
It is shown that every $\mathbf{H} \mathfrak{F}$-group $G$ of type $\mathrm{FP}_{\infty}$ admits a finite dimensional $G$-CWcomplex $X$ with finite stabilizers and with the additional property that for each finite subgroup $H$, the fixed point subspace $X^{H}$ is contractible. This establishes conjecture (5.1.2) of [9]. The construction of $X$ involves joining a family of spaces parametrized by the poset of non-trivial finite subgroups of $G$ and ultimately relies on the theorem of Cornick and Kropholler that if $M$ is a $\mathbb{Z} G$-module which is projective as a $\mathbb{Z} H$-module for all finite $H \leq G$ then $M$ has finite projective dimension.
\end{abstract}

Mathematics Subject Classification (1991). Primary 20J05, 18G20; Secondary 55J05

Keywords. Group, cohomological dimension, classifying space, join, poset

\section{Introduction}

It is well known that a discrete group $G$ has finite cohomological dimension if and only if there is a finite dimensional Eilenberg-Mac Lane space, $K(G, 1)$, or equivalently, a finite dimensional contractible $G$-CW-complex $E G$ on which $G$ acts freely. Groups of finite cohomological dimension are necessarily torsion-free, so that for groups with torsion the advantages of a finite dimensional Eilenberg-Mac Lane space are lost. For this reason it is often convenient to allow $G$-CW-complexes on which $G$ is not required to act freely, but where there can be non-trivial finite isotropy groups. Associated to any group $G$ there is a $G$-CW-complex $\underline{E} G$ with the following properties.

- Every cell stabilizer is finite; and

- for each finite subgroup $H$ of $G$, the fixed point subcomplex $\underline{E} G^{H}$ is contractible.

Moreover, it is not hard to show that any two models of an $\underline{E} G$ are $G$-homotopy equivalent. Our interest in $\underline{E} G$ lies in the fact that for many non-torsion-free groups $G$ there is nevertheless a finite dimensional model for $\underline{E} G$ : for example this applies to all arithmetic groups and all polycyclic-by-finite groups. We refer the reader to Brown's book [4] for general information about cohomological dimension. 
Spaces akin to our $\underline{E} G$ have been considered for compact transformation groups, see [7] for example, but we include here a brief self-contained explanation for discrete groups.

If a group $G$ has an $\underline{E} G$ of finite dimension $D$ then there are a number of homological consequences, for example it can be shown that such a group has rational cohomological dimension at most $D$ and that the integral homology groups $H_{n}(G)$ are torsion for $n>D$. Therefore it is natural to ask

Question 1.1. Is there a simple algebraic characterization of those groups $G$ which admit a finite dimensional $\underline{E} G$ ?

Here, we have been motivated by the conjectures in Section 5.1 of [9] which concern properties of the class $\mathbf{H} \mathfrak{F}$ of hierarchically decomposable groups, and to set the stage, we begin by reviewing this class. First, given any class $\mathfrak{X}$ of groups, we write $\mathbf{H}_{1} \mathfrak{X}$ for the class of groups $G$ which admit a finite dimensional contractible $G$-CW-complex $X$ with cell stabilizers in $\mathfrak{X}$, and we write $\mathbf{H} \mathfrak{X}$ for the smallest $\mathbf{H}_{1}$-closed class containing $\mathfrak{X}$. In other words, $\mathbf{H} \mathfrak{X}$ is the smallest class $\mathfrak{Y}$ containing $\mathfrak{X}$ such that $\mathbf{H}_{1} \mathfrak{Y}=\mathfrak{Y}$. Let $\mathfrak{F}$ denote the class of finite groups. Then it is clear that every group $G$ possessing a finite dimensional $\underline{E} G$ belongs to $\mathbf{H}_{1} \mathfrak{F}$, and it is conceivable that every $G$ in $\mathbf{H}_{1} \mathfrak{F}$ admits a finite dimensional $\underline{E} G$ but we do not know this. Results concerning $\mathbf{H} \mathfrak{F}$ are often proved by transfinite induction: for each ordinal $\alpha$, one can define classes $\mathbf{H}_{\alpha} \mathfrak{F}$ by setting $\mathbf{H}_{0} \mathfrak{F}=\mathfrak{F}$, $\mathbf{H}_{\alpha} \mathfrak{F}=\mathbf{H}_{1}\left(\mathbf{H}_{\alpha-1} \mathfrak{F}\right)$ if $\alpha$ is a successor, and $\mathbf{H}_{\alpha} \mathfrak{F}=\cup_{\beta<\alpha} \mathbf{H}_{\beta} \mathfrak{F}$ if $\alpha$ is a limit ordinal. A group belongs to $\mathbf{H} \mathfrak{F}$ if and only if it belongs to $\mathbf{H}_{\alpha} \mathfrak{F}$ for some $\alpha$.

A similar class of groups can be defined in which we include the additional hypothesis that all groups act cocompactly. We write $\mathbf{K}_{1} \mathfrak{X}$ for the class of groups $G$ which admit a finite dimensional contractible cocompact $G$-CW-complex with stabilizers in $\mathfrak{X}$, and we write $\mathbf{K} \mathfrak{X}$ for the smallest $\mathbf{K}_{1}$-closed class containing $\mathfrak{X}$. The strongest conjecture (5.1.1) of [9] asserted that every $\mathbf{H} \mathfrak{F}$-group of type $\mathrm{FP}_{\infty}$ belongs to $\mathbf{K}_{1} \mathfrak{F}$, but this is now known to be false on account of the beautiful examples [2] of Bestvina and Brady of groups of type FP which are not finitely presented: all groups of type $\mathrm{FP}$ belong to $\mathbf{H}_{1} \mathfrak{F}$ and a fortiori have type $\mathrm{FP}_{\infty}$, while all $\mathbf{K}_{1} \mathfrak{F}$-groups and indeed all $\mathbf{K} \mathfrak{F}$-groups are finitely presented. Here we settle conjecture (5.1.2) of [9] affirmatively, namely that every $\mathbf{H} \mathfrak{F}$-group of type $\mathrm{FP}_{\infty}$ belongs to $\mathbf{H}_{1} \mathfrak{F}$. More precisely, we prove

Theorem A. Let $G$ be an $\mathbf{H} \mathfrak{F}$-group of type $\mathrm{FP}_{\infty}$. Then there is a finite dimensional model for $\underline{E} G$.

It should be noted that the hypothesis that $G$ belongs to $\mathbf{H} \mathfrak{F}$ cannot be dropped here: the discovery [5] of Brown and Geoghegan that Thompson's group

$$
\left\langle x_{0}, x_{1}, x_{2}, \ldots, \mid x_{i}^{-1} x_{j} x_{i}=x_{j+1},(i<j)\right\rangle
$$

is of type $\mathrm{FP}_{\infty}$ confirms it as an example of an infinite dimensional torsion-free 
$\mathrm{FP}_{\infty}$ group which therefore cannot possess a finite dimensional $\underline{E} G$.

\section{Notation and strategy}

Throughout, we write $\Lambda(G)$ for the poset of non-trivial finite subgroups of a group $G$. The $G$-simplicial complex $|\Lambda(G)|$ determined by this poset will be our basic building block for models of $\underline{E} G$. An $n$-simplex in $|\Lambda(G)|$ is determined by each strictly increasing chain

$$
H_{0}<H_{1}<\cdots<H_{n}
$$

of $n+1$ non-trivial finite subgroups. The action of $G$ by conjugation on the set of finite subgroups induces an action of $G$ on $|\Lambda(G)|$ so that the stabilizer of a simplex is an intersection of normalizers: in the case of the simplex determined by the chain of subgroups above the stabilizer is

$$
\bigcap_{i=0}^{n} N_{G}\left(H_{i}\right) \text {. }
$$

In view of theorem 3.4.1 of [9], the results here provide no new information in the torsion-free case, but they make a dramatic improvement in understanding the non-torsion-free case. The poset $\Lambda(G)$ is the crucial new ingredient which does not appear in the earlier algebraic studies of $\mathbf{H} \mathfrak{F}$-groups of type $\mathrm{FP}_{\infty}$, and the remarkable but simple fact which makes $\Lambda(G)$ so useful in this context is as follows:

Lemma 2.1. For any group $G$ and any non-trivial finite subgroup $H$ of $G$, the fixed point complex $|\Lambda(G)|^{H}$ is contractible.

Proof. It suffices to prove that the poset $\Lambda(G)^{H}$ is conically contractible in Quillen's sense (see section 1.5 of [12]). Note that if $K \in \Lambda(G)^{H}$ then $K$ is normalized by $H$ and $H K$ is a finite subgroup of $G$. Let $f: \Lambda(G)^{H} \rightarrow \Lambda(G)^{H}$ be the function defined by $f(K)=H K$. Then, for all $K \in \Lambda(G)^{H}$, we have

$$
K \leq f(K) \geq H
$$

so that $\Lambda(G)^{H}$ is conically contractible. As Quillen points out, this implies that the self-map of $\left|\Lambda(G)^{H}\right|=|\Lambda(G)|^{H}$ induced by $f$ is homotopic both to the constant map with value $H$ and to the identity map. The lemma follows.

Happily, in view of our goal to construct a finite dimensional $\underline{E} G$, we may state the following lemma proved in [8].

Lemma 2.2. If $G$ is an $\mathbf{H} \mathfrak{F}$-group of type $\mathrm{FP}_{\infty}$ then there is a bound on the orders of the finite subgroups of $G$ and in particular $|\Lambda(G)|$ is finite dimensional. 
Our proof of Theorem A uses induction on $\operatorname{dim}|\Lambda(G)|$, taking advantage of the obvious fact that if $H$ is a non-trivial finite subgroup of $G$ then $\operatorname{dim}\left|\Lambda\left(N_{G}(H) / H\right)\right|$ $<\operatorname{dim}|\Lambda(G)|$. However, it is not known that the $\mathrm{FP}_{\infty}$ property is inherited by normalizers of finite subgroups, and so in order to make the inductive step of the proof work, we shall weaken the inductive hypothesis and make use of a subgroup closed property. This is most conveniently expressed in terms of the $G$-operator ring $B(G, \mathbb{Z})$ of bounded functions from $G$ to $\mathbb{Z}$. This ring was first applied in [10] where it was shown to be free as a $\mathbb{Z} H$-module for each finite subgroup of $G$. The following is a result of Cornick and Kropholler [6].

Proposition 2.3. If $G$ is an $\mathbf{H} \mathfrak{F}$-group of type $\mathrm{FP}_{\infty}$ then $B(G, \mathbb{Z})$ has finite projective dimension.

The property proj. $\operatorname{dim}_{\mathbb{Z} G} B(G, \mathbb{Z})<\infty$ is easily seen to be inherited by subgroups, and we shall make considerable use of this fact.

Theorem $\mathrm{A}$ is proved by showing that if $G$ is any $\mathbf{H} \mathfrak{F}$-group such that $d=$ $\operatorname{dim}|\Lambda(G)|<\infty$ and $m=$ proj. $\operatorname{dim}_{\mathbb{Z} G} B(G, \mathbb{Z})<\infty$ then there is an $\underline{E} G$ with finite dimension bounded by a function of $d$ and $m$ alone. Our argument leads to an explicit formula bounding the dimension of $\underline{E} G$ and the induction is easier to describe if we make this bound clear at the outset.

Definition 2.4. Let $\mathbb{N}^{-}$denote the set of integers $d \geq-1$. We write $D=D(d, m)$ for the function $D: \mathbb{N}^{-} \times \mathbb{N} \rightarrow \mathbb{N}$ defined as follows:

- $D(d, 0)=0$ for all $d \geq-1$;

- $D(d, 1)=1$ for all $d \geq-1$;

- $D(-1,2)=3$;

- $D(d, 2)=2^{d} \cdot 7-1$ for all $d \geq 0$;

- $D(d, m)=2^{d+1}(m+1)-1$ for all $d \geq-1$ and $m \geq 3$.

Theorem B. If $G$ is an $\mathbf{H} \mathfrak{F}$-group such that both $d=\operatorname{dim}|\Lambda(G)|$ and $m=$ proj. $\operatorname{dim}_{\mathbb{Z} G} B(G, \mathbb{Z})$ are finite then there is a model for $\underline{E} G$ of dimension $\leq D(d, m)$. (Here we use the convention that $\operatorname{dim}|\Lambda(G)|=-1$ means $G$ is torsion-free.)

In view of Lemma 2.2 and Proposition 2.3, Theorem A is an immediate consequence of Theorem B. In addition we also have

Corollary B. If $G$ is an $\mathbf{H}_{1} \mathfrak{F}$-group such that $|\Lambda(G)|$ is finite dimensional then there is a finite dimensional $\underline{E} G$.

Proof. Let $X$ be a finite dimensional contractible $G$-CW-complex with finite isotropy. Recall that $B(G, \mathbb{Z})$ is free as a $\mathbb{Z} H$-module for all finite subgroups $H$ of $G$ (see [10]). Therefore, tensoring the augmented cellular chain complex $C_{*}(X) \rightarrow \mathbb{Z}$ over $\mathbb{Z}$ with $B(G, \mathbb{Z})$ yields a projective resolution $B(G, \mathbb{Z}) \otimes C_{*}(X) \rightarrow B(G, \mathbb{Z})$ 
of length equal to the dimension of $X$. Thus the hypotheses of Theorem B are satisfied.

Note that all groups of finite virtual cohomological dimension belong to $\mathbf{H}_{1} \mathfrak{F}$ and obviously have finite dimensional $\Lambda$, so the Corollary includes this classical case.

However, there are also many groups in $\mathbf{H}_{1} \mathfrak{F}$, and indeed groups with finite dimensional $\underline{E}$ for which $\Lambda$ is infinite dimensional. For example it can be shown that the wreath product of a non-trivial finite group by an infinite cyclic group has a 3-dimensional $\underline{E}$ even though it has an infinite locally finite subgroup. Examples of these kinds suggest that some new ideas are required to address Question 1.1.

The remainder of the paper is devoted to proving Theorem B. The theorem demands the construction of a certain CW-complex, and there are three methods of construction which we use. First we use the standard procedure where cells are added to a space to kill homology by appealing to the Hurewicz Theorem. Secondly we use the simplicial complex naturally associated to a poset and thirdly we use the join construction. In Section 8 we introduce a general method for joining a family of spaces parametrized by a poset: on the one hand, this reduces to the standard join of spaces when the poset is finite and totally ordered, and on the other hand it specializes to the ordinary simplicial realization of the poset in case the space associated to each poset element is a point.

\section{G-CW-complexes for discrete groups G}

Let $G$ be a discrete group. A $G$-CW-complex consists of a Hausdorff space $X$ together with an action of $G$ by self-homeomorphisms and a filtration $X^{0} \subseteq X^{1} \subseteq$ $X^{2} \subseteq \cdots \subseteq X$ by $G$-subspaces such that the following axioms hold:

1. Each $X^{n}$ is closed in $X$.

2. $\bigcup_{n \in \mathbb{N}} X^{n}=X$.

3. $X^{0}$ is a discrete subspace of $X$.

4. For each $n \geq 1$ there is a discrete $G$-set $\Delta_{n}$ together with $G$-maps $f: S^{n-1} \times$ $\Delta_{n} \rightarrow X^{n-1}$ and $\widehat{f}: B^{n} \times \Delta_{n} \rightarrow X^{n}$ such that the following diagram is a pushout diagram:

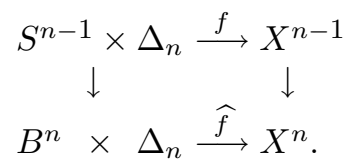

5. A subspace $Y$ of $X$ is closed if and only if $Y \cap X^{n}$ is closed for each $n \geq 0$.

Here, we write $S^{n-1}$ and $B^{n}$ for the standard unit sphere and unit ball in Euclidean $n$-space, and the vertical maps in the diagram are inclusions. It is useful to adopt the conventions $X^{-1}=\emptyset$ and $\Delta_{0}=X^{0}$. Then for all $n \geq 0$, the $n$th cellular chain 
group $C_{n}(X)$ can be defined to be the $n$th singular homology of the pair $X^{n}, X^{n-1}$, and it follows from the Eilenberg-Steenrod Axioms that this is isomorphic as a $G$ module to the permutation module $\mathbb{Z} \Delta_{n}$ determined by $\Delta_{n}$. In effect, $C_{n}(X)$ is the free abelian group on the $G$-set of $n$-cells in $X$.

A $G$-CW-complex $X$ is said to be finite dimensional if and only if $X^{n}=X$ for some $n$, in which case the dimension is the least $n \geq-1$ for which this happens. For the finite dimensional case, Axiom 5 is redundant.

\section{Joins}

The most important construction we shall need is that of join. For convenience we include a definition here. Further discussion can be found in [7], chapter I, section 6 . For each $n \geq 0$ let $\sigma^{n}$ denote the standard $n$-simplex in $\mathbb{R}^{n+1}$ :

$$
\sigma^{n}=\left\{\left(t_{0}, \ldots, t_{n}\right) \mid \sum t_{i}=1, t_{i} \geq 0\right\} .
$$

Given CW-complexes $X_{0}, \ldots, X_{n}$, the join $X_{0} * \cdots * X_{n}$ is defined to be the identification space

$$
\left(\sigma^{n} \times X_{0} \times \cdots \times X_{n}\right) / \sim
$$

where

$$
\left(t_{0}, \ldots, t_{n} ; x_{0}, \ldots, x_{n}\right) \sim\left(t_{0}^{\prime}, \ldots, t_{n}^{\prime} ; x_{0}^{\prime}, \ldots, x_{n}^{\prime}\right)
$$

if and only if for each $i$, either $\left(t_{i}, x_{i}\right)=\left(t_{i}^{\prime}, x_{i}^{\prime}\right)$ or $t_{i}=t_{i}^{\prime}=0$. Note that if $X_{i}$ has dimension $d_{i}$ then the join has dimension $n+\sum d_{i}$. Moreover, if $G$ is a group and the $X_{i}$ are $G$-CW-complexes then the join inherits a $G$-CW-structure.

Now suppose that $X$ and $Y$ are models for $\underline{E} G$. The join construction provides one way of establishing that $X$ and $Y$ are $G$-homotopy equivalent. To see this we shall appeal to the following fundamental result (proposition II 2.7 of [7]):

Proposition 4.1. Let $f: X \rightarrow Y$ be a $G$-map between $G$-CW-complexes such that for all subgroups $H$ of $G$, the induced map $f^{H}: X^{H} \rightarrow Y^{H}$ between the $H$-fixed point subspaces is a homotopy equivalence. Then $f$ is a G-homotopy equivalence.

From this it is immediate that any $G$-map between models of $\underline{E} G$ must be a $G$-homotopy equivalence. Now if $X$ and $Y$ are arbitrary models of $\underline{E} G$ then it is easy to verify that $X * Y$ is again a model for $\underline{E} G$, and that the natural maps $X \rightarrow X * Y$ and $Y \rightarrow X * Y$ are $G$-homotopy equivalences. Alternatively, one could observe that the product $X \times Y$ is an $\underline{E} G$ and that the projections $X \times Y \rightarrow X$ and $X \times Y \rightarrow Y$ are $G$-homotopy equivalences. Either way, it follows that $X$ and $Y$ are $G$-homotopy equivalent.

One of the simplest ways of taking advantage of joins is to form the join of $X$ with the discrete $G$-space $G$ itself. This device makes a space more highly 
connected while at the same time adjoins only free orbits of cells of dimensions no more than one greater than those in $X$.

Lemma 4.2. Let $X$ be a $G$-CW-complex. If $X$ has dimension $n<\infty$ then $X * G$ has dimension $n+1$. If $X$ is acyclic then $X * G$ is contractible. If $X$ is n-connected, for some $n \geq-1$ then $X * G$ is $(n+1)$-connected.

Remark 4.3. This lemma has the useful consequence that the definition of the operation $\mathbf{H}_{1}$ is unaffected whether it is stated in terms of acyclic spaces or contractible spaces. If $X$ is a finite dimensional acyclic $G$-CW-complex with stabilizers in some class $\mathfrak{X}$ which contains the trivial group then $X * G$ is a contractible space, still finite dimensional, and the stabilizers again belong to $\mathfrak{X}$.

Lemma 4.4. Let $Y$ be a $G$-CW-complex of dimension at most $n \leq \infty$. Then $Y$ can be embedded into an $n$-dimensional $G$-CW-complex $\widetilde{Y}$ which is $(n-1)$ connected in such a way that $G$ acts freely outside $Y$.

Proof. We can take $\widetilde{Y}$ to be the $n$-skeleton of $Y * \underbrace{G * \cdots * G}_{n}$. Lemma 4.2 shows that this has the desired properties.

\section{Adding cells using the Hurewicz theorem}

Proposition 5.1. Let $X$ be an $(n-1)$-connected $n$-dimensional $G$-CW-complex, where $n \geq 2$. Let $\phi: F \rightarrow H_{n}(X)$ be a surjective $\mathbb{Z} G$-module map from a free $\mathbb{Z} G$ module $F$ to the nth homology of $X$. Then $X$ can be embedded into an n-connected $(n+1)$-dimensional $G$ - $C W$-complex $X^{\prime}$ so that $G$ acts freely outside $X$ and there is a short exact sequence

$$
0 \rightarrow H_{n+1}\left(X^{\prime}\right) \rightarrow F \stackrel{\phi}{\rightarrow} H_{n}(X) \rightarrow 0 .
$$

Proof. We briefly sketch the proof. Fix one choice $e$ of generator for the $n$th homology $H_{n}\left(S^{n}\right)$ of the $n$-sphere. Given a map $f: S^{n} \rightarrow X$, there is an induced map $f_{*}$ in homology, and hence we obtain a class $f_{*}(e) \in H_{n}(X)$ which depends only on the homotopy class of $f$. Thus there is a well defined $G$-map

$$
\left[S^{n}, X\right] \rightarrow H_{n}(X)
$$

from the set of free homotopy classes of maps $S^{n} \rightarrow X$ to the $n$th homology of $X$. It follows from the Hurewicz theorem that this map is a bijection. Now let $B$ be a basis of $F$ as a $\mathbb{Z} G$-module. For each $b \in B$, choose a map $\tilde{b}: S^{n} \rightarrow X$ so that $\tilde{b}_{*}(e)=\phi b$. Let $\Delta$ denote the $G$-set spanned by $B$. Thus $\Delta$ is a basis of $F$ as 
free abelian group and $F$ is isomorphic to the permutation module $\mathbb{Z} \Delta$. Since $G$ acts freely (i.e. semi-regularly) on $\Delta$, we can define a $G$-map $f: S^{n} \times \Delta \rightarrow X$ by $f(z, b g):=(\tilde{b} z) g$. The space $X^{\prime}$ is now defined to be the pushout:

$$
\begin{array}{r}
S^{n} \times \Delta \stackrel{f}{\longrightarrow} X \\
\downarrow \\
B^{n+1} \times \Delta \longrightarrow X^{\prime} .
\end{array}
$$

Corollary 5.2. Let $X$ be as above. If $H_{n}(X)$ has projective dimension $m<\infty$ over $\mathbb{Z} G$ then $X$ can be embedded into a contractible $G$-CW-complex of dimension $n+m+1$ in such a way that $G$ acts freely outside $X$.

Proof. Choose a free resolution $F_{*} \rightarrow H_{n}(X)$ of length $m$. The result follows by induction on $m$ using Lemma 5.1 at the inductive step.

\section{Homological matters}

Groups and modules of type $\mathrm{FP}_{\infty}$ have been studied in a series of papers by Cornick and Kropholler, and a number of algebraic results have been established. Here we need the following variation on one of these results:

Proposition 6.1. Let $G$ be an $\mathbf{H} \mathfrak{F}$-group and let $M$ be a $\mathbb{Z} G$-module. If $M$ is projective as a $\mathbb{Z} H$-module for all finite subgroups $H$ of $G$ then proj. $\operatorname{dim}_{\mathbb{Z} G} M \leq$ proj. $\operatorname{dim}_{\mathbb{Z} G} B(G, \mathbb{Z})$.

Proof. This is a special case of a result of Cornick and Kropholler which has been recorded as theorem 5.7 of [1].

This result will enable us to build a contractible $G$-CW-complex from some more basic $G$-CW-complex.

Proposition 6.2. Let $G$ be any group, and let $Y$ be a n-dimensional $G-C W$ complex which is $(n-1)$-connected, $(n \geq 0)$. Suppose that $Y^{H}$ is contractible for all non-trivial finite subgroups $H$ of $G$. Then the $n$th reduced homology group $\widetilde{H}_{n}(Y)$ is projective as a $\mathbb{Z} H$-module for all finite subgroups $H$ of $G$.

Proof. Let $H$ be a non-trivial finite subgroup of $G$. Let $Y^{\prime}$ be the subcomplex of $Y$ comprising those cells which are fixed by a non-trivial element of $H$. Then $Y^{\prime}$ can be expressed as the union

$$
\bigcup_{K} Y^{K}
$$


where $K$ runs through the non-trivial subgroups of $H$. Since the family of $Y^{K}$ is closed under intersections and comprises contractible spaces, it follows that $Y^{\prime}$ is contractible. The inclusion of $Y^{\prime}$ in $Y$ induces a short exact sequence

$$
0 \rightarrow \widetilde{C}_{*}\left(Y^{\prime}\right) \rightarrow \widetilde{C}_{*}(Y) \rightarrow C_{*}\left(Y, Y^{\prime}\right) \rightarrow 0
$$

of chain complexes of $\mathbb{Z} H$-modules. (Here, the notation $\widetilde{C}$ is used to indicate the augmented cellular chain complex.) Since $Y^{\prime}$ is contractible, we have $\widetilde{H}_{i}(Y) \cong$ $H_{i}\left(Y, Y^{\prime}\right)$ for each $i$, where $\widetilde{H}$ denotes reduced homology, and hence $H_{n}\left(Y, Y^{\prime}\right)$ is the only non-zero relative homology group and we have an exact sequence

$$
0 \rightarrow H_{n}\left(Y, Y^{\prime}\right) \rightarrow C_{n}\left(Y, Y^{\prime}\right) \rightarrow \cdots \rightarrow C_{0}\left(Y, Y^{\prime}\right) \rightarrow 0 .
$$

Now $H$ acts freely outside $Y^{\prime}$, and this implies that each relative chain group $C_{i}\left(Y, Y^{\prime}\right)$ is free as a $\mathbb{Z} H$-module. Therefore the above sequence splits and we conclude that

$$
H_{n}\left(Y, Y^{\prime}\right) \oplus C_{n-1}\left(Y, Y^{\prime}\right) \oplus \ldots \cong C_{n}\left(Y, Y^{\prime}\right) \oplus C_{n-2}\left(Y, Y^{\prime}\right) \oplus \ldots
$$

so that $\widetilde{H}_{n}(Y) \cong H_{n}\left(Y, Y^{\prime}\right)$ is projective over $\mathbb{Z} H$ as required.

In practice, the assumption that $Y$ is $(n-1)$-connected in this Proposition is not too restrictive, because we can build highly connected spaces from others by using the join construction, as in Lemma 4.4.

\section{Group theoretic properties which are inherited by Weyl groups}

Fix any group $G$, and let $H$ be a finite subgroup. Here we gather three results concerning properties of $G$ which are inherited by the "Weyl group" $W:=N_{G}(H) / H$. The first two are needed later, while the third is included for comparison.

Lemma 7.1. If $G$ is an $\mathbf{H} \mathfrak{F}$-group then so is $W$.

Proof. Since $\mathbf{H} \mathfrak{F}$ is a subgroup closed class we may assume without loss of generality that $H$ is normal in $G$. There is nothing to prove if $G$ is finite, so we may assume that $G$ belongs to $\mathbf{H}_{\alpha} \mathfrak{F}$ for some ordinal $\alpha>0$ and that the result is true for groups in any $\mathbf{H}_{\beta} \mathfrak{F}$ with $\beta<\alpha$. Let $X$ be a finite dimensional contractible $G$-CWcomplex in which each cell stabilizer $G_{x}$ belongs to $\mathbf{H}_{\beta} \mathfrak{F}$ for some $\beta<\alpha$. Now $X / H$ is acyclic, by theorem III 5.4 of [3] and may be regarded as a $G / H$-CW-complex. Moreover, each cell stabilizer in this action is of the form $G_{x} H / H \cong G_{x} / G_{x} \cap H$ for some cell $x$ of $X$, and hence by induction (and Remark 4.3), each stabilizer belongs to $\mathbf{H F}$. Therefore so does $G / H$. 
For the second result, we need the following preparatory Lemma.

Lemma 7.2. Let $P$ be a projective $\mathbb{Z} G$-module. Then $P^{H}$ is a projective $\mathbb{Z} W$ module.

Proof. Let $\widehat{h}:=\sum_{h \in H} h$. Then the map $\mathbb{Z} G^{H} \rightarrow \mathbb{Z}[H \backslash G]$ defined by $\widehat{h} g \mapsto H g$ is a bijective $\mathbb{Z} W$-map and this establishes the result when $P$ is equal to $\mathbb{Z} G$. The general case clearly follows.

Lemma 7.3. proj. $\operatorname{dim}_{\mathbb{Z} W} B(W, \mathbb{Z}) \leq$ proj. $\operatorname{dim}_{\mathbb{Z} G} B(G, \mathbb{Z})$.

Proof. Let $m=$ proj. $\operatorname{dim}_{\mathbb{Z} G} B(G, \mathbb{Z})$ and let $N=N_{G}(H)$. The $\mathbb{Z} N$-module inclusion of $B(N, \mathbb{Z})$ in $B(G, \mathbb{Z})$ defined by extending functions to be zero on the complement of $N$ is split by the restriction map $B(G, \mathbb{Z}) \rightarrow B(N, \mathbb{Z})$. Therefore $B(N, \mathbb{Z})$ has projective dimension at most $m$ over $\mathbb{Z} N$. Let

$$
0 \rightarrow P_{m} \rightarrow \cdots \rightarrow P_{0} \rightarrow B(N, \mathbb{Z}) \rightarrow 0
$$

be a projective resolution. Since $B(N, \mathbb{Z})$ is free as a $\mathbb{Z} H$-module, [10], this sequence splits over $\mathbb{Z} H$. Therefore taking $H$-fixed points preserves exactness of the sequence and produces a resolution of $B(N, \mathbb{Z})^{H}=B(W, \mathbb{Z})$ of the same length. Lemma 7.2 shows that this is a $\mathbb{Z} W$-projective resolution as required.

Lemma 7.4. If there exists a finite dimensional $\underline{E} G$ then there is a finite dimensional $\underline{E} W$.

Proof. Let $X$ be a finite dimensional model for $\underline{E} G$. Then the $H$-fixed point subcomplex $X^{H}$ is a model for $\underline{E} W$.

\section{A general construction}

Suppose given a map $f: X \rightarrow \Lambda$ where $\Lambda$ is a discrete poset and $X$ is a CWcomplex. For each $\lambda$ in $\Lambda$, let $X(\lambda)$ denote the preimage of $\lambda$. We describe here a general procedure for joining the spaces $X(\lambda)$ together to make a new space $|f|$. In effect $|f|$ is similar to the usual simplicial realization of $\Lambda$ except that each face of $|\Lambda|$ is replaced by the join of the spaces $X(\lambda)$ associated to the vertices of that face. In this way we shall be able to arrange, in the case where there is a group acting, that $|f|$ has much smaller isotropy groups than $|\Lambda|$.

Let $\Lambda^{n}$ denote the set of strictly increasing sequences $\underline{\lambda}:=\lambda_{0}<\cdots<\lambda_{n}$ of elements of $\Lambda$, and let $X(\underline{\lambda})=X\left(\lambda_{0}\right) \times \cdots \times X\left(\lambda_{n}\right)$. For $0 \leq i \leq n$, let $d_{i}$ denote the standard face map from $\sigma^{n-1}$ to $\sigma^{n}$. For $\underline{\lambda} \in \Lambda^{n}$, let $\underline{\lambda}^{(i)}$ denote the sequence in $\Lambda^{n-1}$ obtained by omitting the $i$ th term of $\underline{\lambda}$ and let $\pi_{i}$ denote the natural 
projection from $X(\underline{\lambda})$ to $X\left(\underline{\lambda}^{(i)}\right)$. We define $|f|$ to be the quotient space

$$
\left(\coprod_{n \in \mathbb{N}} \coprod_{\underline{\lambda} \in \Lambda^{n}} \sigma^{n} \times X(\underline{\lambda})\right) / \sim,
$$

where, for each $n \in \mathbb{N}, \underline{\lambda} \in \Lambda^{n}, v \in \sigma^{n-1}$ and $x \in X(\underline{\lambda})$ we make the identifications

$$
\left(d_{i} v, x\right) \sim\left(v, \pi_{i} x\right)
$$

for $0 \leq i \leq n$.

Example 8.1. If $X=\Lambda$ and $f: X \rightarrow \Lambda$ is the identity map then $|f|$ is simply the usual simplicial realization $|\Lambda|$ of the poset $\Lambda$.

Example 8.2. If $\Lambda$ is the set $\{0,1, \ldots, n\}$ with the usual ordering then $|f|$ is the join $X(0) * \cdots * X(n)$.

Lemma 8.3. Given two maps $f: X \rightarrow \Lambda$ and $f^{\prime}: X^{\prime} \rightarrow \Lambda$ and a map $h: X \rightarrow X^{\prime}$ so that $f^{\prime} \circ h=f$, there is an induced $\operatorname{map} h_{*}:|f| \rightarrow\left|f^{\prime}\right|$.

Proof. For each $\underline{\lambda}, h$ induces a map $X(\underline{\lambda}) \rightarrow X^{\prime}(\underline{\lambda})$ compatible with identifications and yielding $h_{*}:|f| \rightarrow\left|f^{\prime}\right|$.

Corollary 8.4. Given $f: X \rightarrow \Lambda$, there is always a natural map $|f| \rightarrow|\Lambda|$ and if each $X(\lambda)$ is non-empty then there exists a map $|\Lambda| \rightarrow|f|$ and $|\Lambda|$ is a retract of $|f|$.

Proof. First apply the Lemma with $X^{\prime}$ being $\Lambda, f^{\prime}$ being the identity map and with $h=f$. If each $X(\lambda)$ is non-empty, then we can choose a map $h: \Lambda \rightarrow X$ so that $h(\lambda) \in X(\lambda)$, and the second part of the Corollary follows by applying the Lemma to this $h$.

Lemma 8.5. Suppose given a homotopy $H: X \times I \rightarrow X^{\prime}$ between two maps $h_{0}, h_{1}$ and maps $f: X \rightarrow \Lambda$ and $f^{\prime}: X^{\prime} \rightarrow \Lambda$ such that for all $t \in I$ and $x \in X$, $f^{\prime}(H(x, t))=f(x)$. Then the induced maps $\left(h_{0}\right)_{*},\left(h_{1}\right)_{*}:|f| \rightarrow\left|f^{\prime}\right|$ are homotopic.

Proof. Let $i_{0}$ and $i_{1}$ denote the inclusions $X \rightarrow X \times I$ given by $x \mapsto(x, 0)$ and $x \mapsto(x, 1)$ respectively, and let $\bar{f}: X \times I \rightarrow \Lambda$ denote the composition of the projection $X \times I \rightarrow X$ with $f$. Our construction is functorial, so that $\left(h_{0}\right)_{*}=H_{*} \circ\left(i_{0}\right)_{*}$ and $\left(h_{1}\right)_{*}=H_{*} \circ\left(i_{1}\right)_{*}$, and so it suffices to prove that $\left(i_{0}\right)_{*}$ and $\left(i_{1}\right)_{*}$ are homotopic maps from $|f|$ to $|\bar{f}|$. Let $j_{0}$ and $j_{1}$ denote the inclusions of $|f|$ into $|f| \times I$ given by $x \mapsto(x, 0)$ and $x \mapsto(x, 1)$ respectively. By construction each $(X \times I)(\underline{\lambda})$ is naturally homeomorphic to $X(\underline{\lambda}) \times I^{n+1}$ and the diagonal map 
$I \rightarrow I^{n+1}$ therefore induces a map $\Delta_{*}:|f| \times I \rightarrow|\bar{f}|$ and we have $\left(i_{0}\right)_{*}=\Delta_{*} \circ j_{0}$ and $\left(i_{1}\right)_{*}=\Delta_{*} \circ j_{1}$. Since $j_{0}$ and $j_{1}$ are obviously homotopic, the result follows.

The following consequence is immediate:

Corollary 8.6. If each $X(\lambda)$ is contractible then the natural map $|f| \rightarrow|\Lambda|$ is a homotopy equivalence.

Our construction is natural, and so behaves well in the presence of a group action:

Lemma 8.7. If a group $G$ acts on $\Lambda$ and $X$ in such a way that $f: X \rightarrow \Lambda$ is a $G$-map, then $|f|$ is a $G$-CW-complex and the natural map $|f| \rightarrow|\Lambda|$ is a $G$-map. Moreover, for any subgroup $H$ of $G$, the $H$-fixed point space $|f|^{H}$ may be naturally identified with $\left|f^{H}\right|$, where $f^{H}: X^{H} \rightarrow \Lambda^{H}$ is the map on $H$-fixed point subspaces induced by $f$.

Proof. For any subgroup $H$ of $G$ there is clearly a natural inclusion $\left|f^{H}\right| \rightarrow|f|^{H}$ and it is enough to observe that this is surjective. Given any element $u \in|f|^{H}$, there is a least $n$ such that it is represented by some $(v, x)$ in one of the contributions $\sigma^{n} \times X(\underline{\lambda})$. Either $n=0$ or $v$ belongs to the interior of $\sigma^{n}$ and in either case it is clear that $x$ must be fixed by $H$.

In practice we shall take advantage of the equivariant form of this construction in the following way. Let $G$ be a group. If $N$ is a subgroup of $G$ and $X$ is an $N$-CW-complex, then we can form the induced space $X \times{ }_{N} G$. This is a quotient space obtained from the Cartesian product $X \times G$ by making the identifications $(x n, g) \sim(x, n g)$ for $x \in X, n \in N, g \in G$. We shall write $x \times g$ for the element of $X \times{ }_{N} G$ represented by $(x, g)$.

Now let $\Lambda$ be a $G$-poset, and let $\Lambda_{0}$ be a set of $G$-orbit representatives for $\Lambda$. Suppose given a $G_{\lambda^{-}}$CW-complex $X(\lambda)$ for each $\lambda \in \Lambda_{0}$. Define $X$ to be the disjoint union:

$$
X:=\coprod_{\lambda \in \Lambda_{0}} X(\lambda) \times_{G_{\lambda}} G
$$

and let $f: X \rightarrow \Lambda$ be defined by $f(x \times g)=\lambda g$ for $x \in X(\lambda)$ and $g \in G$. Our construction then yields a $G$-map $|f| \rightarrow|\Lambda|$.

\section{The proof of Theorem B}

Let $G$ be an $\mathbf{H} \mathfrak{F}$-group such that both

$$
d=\operatorname{dim}|\Lambda(G)|
$$


and

$$
m=\text { proj. } \operatorname{dim}_{\mathbb{Z} G} B(G, \mathbb{Z})
$$

are finite. We construct an $\underline{E} G$ of dimension $\leq D(d, m)$ where $D$ is the function introduced in Definition 2.4. For $m \geq 2$ the proof is an induction on $d$, although the case $m=2$ has to be treated in a slightly different way from the cases $m \geq 3$. For $m \leq 1$ there are direct proofs. The base of the induction when $d=-1$ can be treated uniformly and we begin with this.

Lemma 9.1. If $d=-1$ then $G$ has an Eilenberg-Mac Lane space $K(G, 1)$ of dimension $\leq D(-1, m)$.

Proof. When $d=-1, G$ is torsion free and so it follows by applying Proposition 6.1 to the trivial module $\mathbb{Z}$ that $G$ has finite cohomological dimension $\leq m$. Hence there is an Eilenberg-Mac Lane space of dimension $m$ if $m \neq 2$ and of dimension 2 or 3 if $m=2$. This proves the Lemma, since by definition, $D(-1, m)=m$ except when $m=2$ when $D(-1, m)=3$.

Of course, if $G$ has a finite dimensional Eilenberg-Mac Lane space then its universal cover serves as an $\underline{E} G$ of the same dimension, and hence this Lemma deals with the case $d=-1$ of Theorem B for arbitrary $m$.

We next consider the cases in which $m$ is large: fix an $m \geq 3$. Assume that $d \geq 0$ and that by induction the conclusion holds for groups with smaller $d$. Let $\Lambda_{0}$ be a set of conjugacy class representatives for the non-trivial finite subgroups of $G$. For each $H \in \Lambda_{0}$ it is clear that $\operatorname{dim}\left|\Lambda\left(N_{G}(H) / H\right)\right|<d$. Moreover $N_{G}(H) / H$ belongs to $\mathbf{H} \mathfrak{F}$ by Lemma 7.1 and $m^{\prime}:=$ proj. $\operatorname{dim}_{\mathbb{Z}\left[N_{G}(H) / H\right]} B\left(N_{G}(H) / H, \mathbb{Z}\right)$ is at most $m$ by Lemma 7.3 . Hence by induction we can choose a model $X(H)$ of $\underline{E}\left(N_{G}(H) / H\right)$ of dimension $\leq D\left(\operatorname{dim}\left|\Lambda\left(N_{G}(H) / H\right)\right|, m^{\prime}\right) \leq D\left(\operatorname{dim}\left|\Lambda\left(N_{G}(H) / H\right)\right|, m\right)$. We can view $X(H)$ as an $N_{G}(H)$-CW-complex with $H$ acting trivially. Following the procedure indicated at the end of Section 8, we define a space $X$ and a map $f: X \rightarrow \Lambda$ by taking $X$ to be the disjoint union

$$
\coprod_{H \in \Lambda_{0}} X(H) \times_{N_{G}(H)} G
$$

and defining $f$ by

$$
f(x \times g)=H^{g}
$$

for $x \in X(H)$ and $g \in G$. Let $Y$ be the geometric realization $|f|$ of $f$ as defined in Section 8. Each totally ordered chain $H_{0}<\cdots<H_{n}$ of non-trivial finite subgroups of $G$ gives rise to a contribution to $|f|$ which is in effect the join $X\left(H_{0}\right) * \cdots * X\left(H_{n}\right)$. It is clear that $\operatorname{dim} X\left(H_{i}\right) \leq D(d-1-i, m)$ for each $i$, and hence, the join 
$X\left(H_{0}\right) * \cdots * X\left(H_{n}\right)$ has dimension at most

$$
\begin{aligned}
n+\sum_{i=0}^{n} D(d-1-i, m) & \leq d+\sum_{i=0}^{d} D(d-1-i, m)=d+\sum_{i=0}^{d}\left(2^{d-i}(m+1)-1\right) \\
& =\left(2^{d+1}-1\right)(m+1)-1=D(d, m)-(m+1) .
\end{aligned}
$$

Therefore

$$
\operatorname{dim} Y \leq D(d, m)-(m+1) .
$$

Moreover, the natural map $Y \rightarrow|\Lambda(G)|$ is a homotopy equivalence by Corollary 8.6. More crucially, for each non-trivial finite subgroup $H$ of $G$ we have $Y^{H}=\left|f^{H}\right| \simeq\left|\Lambda(G)^{H}\right|=|\Lambda(G)|^{H}$ by Lemma 8.7, and now Corollary 8.6 together with Lemma 2.1 show that $Y^{H}$ is contractible. Also, $G$ acts on $Y$ with finite isotropy. Applying Proposition 4.4 with $n=\max \{\operatorname{dim} Y, 2\}, Y$ is embedded into a $G$-CW-complex $\widetilde{Y}$ in such a way that $G$ acts freely outside $Y$ and so that $\widetilde{Y}$ is $n$-dimensional and $(n-1)$-connected. The choice of $n$ guarantees that $\widetilde{Y}$ is at least simply connected. Note that for any $d \geq 0, D(d, m)-(m+1) \geq D(0, m)-(m+1)=$ $m \geq 3$, and so $\operatorname{dim} \tilde{Y}=n \leq D(d, m)-(m+1)$. Moreover, since $Y$ and $\tilde{Y}$ have the same singular part, it follows that $\tilde{Y}$ inherits the property that the fixed point sets of non-trivial finite subgroups are contractible. Therefore, by Proposition 6.2, $H_{n}(\widetilde{Y})$ is projective as a $\mathbb{Z} H$-module for all finite subgroups $H$ of $G$, and hence by Proposition 6.1 , it has projective dimension $\leq m$. Using Proposition 5.2, we can add free orbits of cells to $\widetilde{Y}$ so that it is embedded into an $\underline{E} G$ of dimension $\operatorname{dim} \widetilde{Y}+m+1 \leq D(d, m)$, as required.

The proof is essentially the same when $m=2$, save that by allowing for the possibility that there are groups of cohomological dimension 2 which do not have 2-dimensional Eilenberg-Mac Lane spaces, the formula for the bound $D(d, m)$ is slightly different. We leave the reader to check that each step of the above argument carries through with the new bound as with the old.

This leaves only the cases $m=0$ and $m=1$. If $m=0$ then $B(G, \mathbb{Z})$ is projective: but it also contains a copy of the trivial module (as the constant functions) and therefore $G$ is finite, in which case we take $\underline{E} G$ to be a one point space. The case $m=1$ is much more interesting. In this case, Talelli has proved [11] that $G$ is the fundamental group of a graph of finite groups by using the Dicks-Dunwoody Almost Stability Theorem. Hence $G$ acts on a tree with finite vertex and edge stabilizers, and this tree serves as a 1-dimensional $\underline{E} G$.

\section{References}

[1] D. J. Benson, Complexity and varieties for infinite groups I, J. Pure Appl. Algebra 193 (1997), 260-287.

[2] M. Bestvina and N. Brady, Morse theory and finiteness properties of groups, Invent. Math. 129 (1997), 445-470. 
[3] G. E. Bredon, Introduction to Compact Transformation Groups, Academic Press 1972.

[4] K. S. Brown, Cohomology of Groups, Graduate Texts in Math. 87, Springer, Berlin 1982.

[5] K. S. Brown and R. Geoghegan, An infinite dimensional $\mathrm{FP}_{\infty}$-group, Invent. Math. 77 (1984), 367-381.

[6] J. Cornick and P. H. Kropholler, Homological finiteness conditions for modules over group algebras, to appear in J. London Math. Soc.

[7] T. tom Dieck, Transformation Groups, de Gruyter Studies in Math. 8 (1987).

[8] P. H. Kropholler, On groups of type FP ${ }_{\infty}$, J. Pure Appl. Algebra 90 (1993), 55-67.

[9] P. H. Kropholler, Hierarchical decompositions, generalized Tate cohomology, and groups of type $\mathrm{FP}_{\infty}$. In: ( A. Duncan, N. Gilbert, and J. Howie, eds.) Proceedings of the Edinburgh Conference on Geometric Group Theory, 1993, Cambridge U. P. Lond. Math. Soc. Lect. Note Ser. 204 (1995), 190-216.

[10] P. H. Kropholler and O. Talelli, On a property of fundamental groups of graphs of finite groups, J. Pure Appl. Algebra 74 (1991), 57-59.

[11] O. Talelli, On groups with $\operatorname{cd}_{\mathbb{Q}} G \leq 1$, J. Pure Appl. Algebra 88 (1993), 245-247.

$[12]$ D. Quillen, Homotopy properties of the poset of non-trivial $p$-subgroups of a group, $A d v$. Math. 28 (1978), 101-128.

Peter H. Kropholler

Mathematical Sciences

Queen Mary

London E1 4NS

e-mail: P.H.Kropholler@qmw.ac.uk

(Received: April 30, 1997)
Guido Mislin Departement Mathematik

ETH Zürich

CH-8092 Zürich

e-mail: mislin@math.ethz.ch 